\title{
Dopamine transporter imaging in neurodegenerative movement disorders: PET vs. SPECT
}

\author{
Vera S. Kerstens ${ }^{1}\left(\mathbb{D} \cdot\right.$ A. Varrone $^{1}[0$ \\ Received: 9 July 2020 / Accepted: 31 August 2020 / Published online: 15 September 2020 \\ (C) The Author(s) 2020
}

\begin{abstract}
Purpose The dopamine transporter (DAT) serves as biomarker for parkinsonian syndromes. DAT can be measured in vivo with single-photon emission computed tomography (SPECT) and positron emission tomography (PET). DAT-SPECT is the current clinical molecular imaging standard. However, PET has advantages over SPECT measurements, and PET radioligands with the necessary properties for clinical applications are on the rise. Therefore, it is time to review the role of DAT imaging with SPECT compared to PET.

Methods PubMed and Web of Science were searched for relevant literature of the previous 10 years. Four topics for comparison were used: diagnostic accuracy, quantitative accuracy, logistics, and flexibility.

Results There are a few studies directly comparing DAT-PET and DAT-SPECT. PET and SPECT both perform well in discriminating neurodegenerative from non-neurodegenerative parkinsonism. Clinical DAT-PET imaging seems feasible only recently, thanks to simplified DAT assessments and better availability of PET radioligands and systems. The higher resolution of PET makes more comprehensive assessments of disease progression in the basal ganglia possible. Additionally, it has the possibility of multimodal target assessment.

Conclusion DAT-SPECT is established for differentiating degenerative from non-degenerative parkinsonism. For further differentiation within neurodegenerative Parkinsonian syndromes, DAT-PET has essential benefits. Nowadays, because of wider availability of PET systems and radioligand production centers, and the possibility to use simplified quantification methods, DAT-PET imaging is feasible for clinical use. Therefore, DAT-PET needs to be considered for a more active role in the clinic to take a step forward to a more comprehensive understanding and assessment of Parkinson's disease.
\end{abstract}

Keywords Parkinson's disease $\cdot$ SPECT $\cdot$ PET $\cdot$ Dopamine transporter $\cdot$ Biomarker

\section{Introduction}

\section{DAT as imaging marker}

Parkinson's disease (PD) is the most common neurodegenerative movement disorder, with approximately 1 out of 1000 people age 50 and older having the disease. Current treatment is solely symptomatic. Considerable efforts and resources are being invested in finding suitable targets for disease-modifying treatments [1]. To be able to assess the effectiveness of a new treatment, a reliable outcome measure

Vera S. Kerstens

vera.kerstens@ki.se

1 Department of Clinical Neuroscience, Centre of Psychiatry Research, Karolinska Institutet and Stockholm Health Care Services, Stockholm, Sweden is needed. Clinical neurological assessment with the (Movement Disorder Society) Unified Parkinson's Disease Rating scale ([MDS-]UPDRS) is currently the most frequently used outcome measure in clinical trials and clinical practice, but is sensitive to interpretation bias. A biomarker directly representing the underlying pathophysiology would be ideal, such as in vivo measurement of misfolded $\alpha$-synuclein, as found in post-mortem PD studies. However, at present, there is no available radioligand for imaging $\alpha$-synuclein [2]. In vivo imaging of dopamine transporter (DAT) availability in the basal ganglia provides a measure of pre-synaptic dopaminergic integrity and function, and, currently, represents a practical indirect biomarker of PD.

The decline of DAT density is secondary to the neurodegeneration of the dopaminergic nerve terminals, but also to functional adaptation of the dopaminergic system; compensatory DAT downregulation might occur in earlier stages 
[3-5] which might explain the relative delay in symptom onset. Since, at symptom onset, already $50 \%$ of dopaminergic terminals are lost, a flooring effect of DAT measurements might occur later on in the disease. Taking these two things into account, the measured DAT decline is most likely non-linear [6, 7], and DAT imaging might be particularly useful in early stages of the disease, both for diagnostic purposes and for assessment of treatment effects.

DAT imaging has been shown to shorten the time to diagnosis of PD, help accurate diagnosis in unclear clinical cases, and, therefore, avoid unnecessary side-effects of dopaminergic treatment in non-neurodegenerative cases [8, 9]. DAT imaging has not been able to differentiate PD from the atypical parkinsonian syndromes (multisystem atrophy, MSA; progressive supranuclear palsy, PSP; and corticobasal disease, CBD). For a comprehensive background overview of the evidence of the role of DAT as a biomarker in neurodegenerative movement disorders, we refer to the recent literature reviews of Maiti and Perlmutter [10], Buchert et al. [11], Palermo and Ceravolo [12].

\section{Current role of DAT-SPECT}

The established radioligand for DAT imaging with singlephoton emission computed tomography (SPECT) is $\left[{ }^{123} \mathrm{I}\right]$ FP-CIT, commercially available in Europe since 2000 and the United States since 2011 (DaTSCAN $^{\circledR} /$ Striascan $^{\mathrm{TM}}$ and DaTscan ${ }^{\circledR}$, respectively). [ ${ }^{123}$ I]FP-CIT SPECT is an established part of the diagnostic guidelines and workflow for patients with clinically uncertain parkinsonism; it performs well in discriminating neurodegenerative from non-neurodegenerative parkinsonism (such as essential tremor or healthy controls), and, in the case of cognitive dysfunction, Lewy Body dementia (DLB) from Alzheimer's dementia [13-15]. Moreover, $\left[{ }^{123} \mathrm{I}\right]$ FP-CIT SPECT has been recently added as an enrichment biomarker in clinical PD studies [16]. Patients with Scans Without Evidence of Dopaminergic Deficit (SWEDD) are thought to have a different disease entity than PD [17]. Their identification with DAT-SPECT is a criterion for exclusion from PD cohorts and helps to select more homogenous PD cohorts in clinical trials.

\section{DAT-PET in the picture: why this review?}

DAT imaging can also be done with positron emission tomography (PET). Although the introduction of solid-state detectors has improved the resolution of SPECT [18], PET still has the advantage of higher sensitivity, higher spatial resolution, and better quantitative accuracy. Higher resolution makes a more detailed assessment of DAT stabilization or decline patterns possible. This would specifically benefit borderline $\left[{ }^{123}\right.$ I]FP-CIT SPECT findings $(<10 \%,[16])$ and evaluation of treatment effects where small changes might already be clinically significant. Several DAT radioligands for PET exist $[19,20]$, including ${ }^{18} \mathrm{~F}$-labeled radioligands $\left[{ }^{18} \mathrm{~F}\right]$ FP-CIT, $\left[{ }^{18} \mathrm{~F}\right]$ LBT-999, and $\left[{ }^{18} \mathrm{~F}\right]$ FE-PE2I. Because of its high selectivity to DAT and its favorable pharmacokinetic properties, $\left[{ }^{18} \mathrm{~F}\right] \mathrm{FE}-\mathrm{PE} 2 \mathrm{I}$ can be currently considered the most accurate option and has been included in the recent EANM guidelines and SNMMI procedure standard for dopaminergic imaging in Parkinsonian syndromes [13].

In the last two decades, DAT-PET imaging has mainly been used as a research tool [6]. More recently, PET-CT systems have become more widely available, and new radiochemistry facilities with standards of good manufacturing practice (GMP) for local radiopharmaceutical productions have been implemented. The number of PET-MRI systems installed worldwide has also increased. In view of these advancements, the question is whether DAT-PET is finally ready for prime time. The aim of this review is to critically address this question by giving an overview of how DAT imaging with PET and SPECT compare in the following four aspects: 1. Diagnostic accuracy, 2. Quantitative accuracy, 3. Logistics, and 4. Flexibility.

\section{Methods}

For this expert review, a non-systematic literature search was done in PubMed and Web of Science based on pre-defined relevant subtopics by the authors. The results were restricted to those published in the last 10 years, because DAT-imaging studies are frequent and resulting insights develop quickly. Other filters used were: English language and human studies only. Relevant articles were selected based on the reading of abstracts and articles.

Forward and backward searches on relevant citations were added, and citations known through expertise by the authors. In cases where the topic has already been comprehensively reviewed, the review is cited instead of the individual studies.

\section{Diagnostic accuracy}

As described in the introduction, $\left[{ }^{123} \mathrm{I}\right] \mathrm{FP}$-CIT SPECT is implemented in diagnostic guidelines on parkinsonism worldwide and has recently been added as an enrichment biomarker for use in clinical PD studies [16]. $\left[{ }^{123}\right.$ I]FP-CIT SPECT achieves high diagnostic accuracy of neurodegenerative parkinsonism, even with just a visual evaluation of the scan [14]. Sensitivity is estimated to be $78-100 \%$ (median $93 \%$ ) and specificity $70-100 \%$ (median $89 \%$ ) for differentiating neurodegenerative versus non-neurodegenerative parkinsonism [11]. For differentiating Alzheimer's disease from Lewy Body dementia, the sensitivity is $\sim 80 \%$ and specificity $\sim 90 \%$ [11]. Of note, the sensitivity and specificity 
were assessed with the diagnosis of a movement disorder specialist as a reference, for lack of the golden reference standard post-mortem verified diagnosis. The high accuracy is the result of the high signal-to-background ratio of the radioligand and the already pronounced DAT deficit at the onset of the first PD motor symptoms. Furthermore, DATSPECT can also assess pre-symptomatic DAT decline in PD patients; significant DAT decline contralateral to the clinically not yet affected side was found compared to healthy controls [21, 22]. DAT-SPECT, thus, performs well for these diagnostic, clinical purposes. A recent study of 28 parkinsonian syndrome (PS) patients and 22 healthy controls (HC) compared DAT availability measurements with both DATSPECT $\left(\left[{ }^{123} \mathrm{I}\right] \mathrm{FP}-\mathrm{CIT}\right)$ and DAT-PET $\left(\left[{ }^{18} \mathrm{~F}\right] \mathrm{FE}-\mathrm{PE} 2 \mathrm{I}\right)$. The discriminative power was similar, with ROC AUC in the putamen for FP-CIT (SUR) 0.891 (95\% CI 0.786-0.994), static $\left[{ }^{18} \mathrm{~F}\right]$ FE-PE2I (SUVR) 0.886 (95\% CI 0.786-0.986), and dynamic $\left[{ }^{18} \mathrm{~F}\right]$ FE-PE2I $\left(B P_{\mathrm{ND}}\right) 0.903(0.81-0.99)$ [23]. A highlighted advantage of PET was its ability to detect cases in which underlying infarctions in the basal ganglia were the reason for lower DAT binding [23]. Their baseline cohort included five SWEDD patients in both [ ${ }^{123}$ I]FP-CIT and $\left[{ }^{18} \mathrm{~F}\right]$ FE-PE2I. For comparing diagnostic accuracy of the two methods, additional studies in larger cohorts of patients are needed. However, considering that the accuracy of DATSPECT is already high, it is unlikely that DAT-PET performs significantly better in this aspect. Similar conclusions were reached in a study comparing DAT-SPECT vs. DATPET with $\left[{ }^{123}\right.$ I]FP-CIT and $\left[{ }^{18}\right.$ F]FP-CIT [24]. Finally, in a meta-analysis on DAT imaging in PD patients investigating the possibility of a bias induced by the imaging method (PET vs SPECT), similar effect sizes were found in 9 PET DAT studies compared to 33 SPECT DAT studies (Hedges $g=3.74$ vs 3.65 , respectively) [6].

With regards to the differential diagnosis between PD and atypical parkinsonian syndromes, studies that compare the two modalities are not available. Although at group level, a more uniform involvement of the striatum has been reported in patients with progressive supranuclear palsy, no reliable distinctive patterns of DAT decline make it possible to individually discriminate patients with atypical parkinsonian syndromes from PD [25-27].

\section{Quantitative accuracy}

Binding potential is the main quantitative outcome measure for neuroreceptor studies with SPECT and PET. In this context, the use of PET radioligands labeled with shortlived isotopes (for example, ${ }^{11} \mathrm{C}$ : half-life $20.33 \mathrm{~min},{ }^{18} \mathrm{~F}$ : $109.77 \mathrm{~min}$ ) makes full quantification and estimation of binding potential possible, whereas longer lived SPECT isotopes (for example, ${ }^{123}$ I: half-life $13.22 \mathrm{~h}$ ) would require several hours of data collection for full quantification.
Furthermore, because of PET's higher spatial resolution and sensitivity, more detailed regional DAT availability can be assessed, and even areas with low DAT density, such as the substantia nigra ( $\mathrm{SN})$, can be included in the assessment, allowing for a more comprehensive assessment of DAT availability in the basal ganglia [28]. A DAT-PET study on 20 patients with early-stage PD and age- and sex-matched healthy controls found a 70\% lower DAT binding in putamen, $40 \%$ in caudate, $36 \%$ in ventral striatum, and $30 \%$ in the SN in PD patients [28]. With the seemingly sparing of dopaminergic neural cell bodies in early stages, the examination of the DAT in SN might be of interest in later stages of the disease. More DAT-PET studies are needed to investigate this further.

Findings on correlations between DAT availability and motor and non-motor symptoms vary, with the majority finding no or weak correlations $[6,29,30]$. This might be because the outcomes applied, use generalized clinical outcomes (such as total UPDRS-III score) and generalized regions in the brain, instead of sub-scales and sub-regions. The availability of striatal dopaminergic markers is classically measured in the main striatal sub-regions, caudate, and putamen. However, the functions within the striatum are not homogeneous and entail different functional sub-regions [31, 32]. An alternative functional subdivision, for example, is the associative, limbic, and sensorimotor subdivision (Fig. 1, [33]), which are thought to represent motor functioning, mood, and cognitive functioning, respectively. The use of the striatal functional subdivisions provides a better way to assess correlations between clinical and behavioral subdomains of Parkinson symptoms with regional DAT deficit $[34,35]$.

\section{Logistics}

Logistically, $\left[{ }^{123} \mathrm{I}\right] \mathrm{FP}-\mathrm{CIT}$ SPECT is currently the most practical method for DAT assessment, since it is already implemented in the clinic. However, PET has some logistical advantages, as well. The shorter half-life of PET radioligands makes it possible for the subject to be examined directly after injection in case of dynamic scanning. In case of static scanning, data are collected within $2 \mathrm{~h}$ of injection in case of commonly used clinical PET radioligands (e.g., amyloid tracers), whereas for clinical SPECT radioligands suchs as $\left[{ }^{123} \mathrm{I}\right] \mathrm{FP}-\mathrm{CIT}$, data collection has to be delayed to 3-6 $\mathrm{h}$ after injection. Hence, the whole PET imaging procedure can be done within a shorter time frame than SPECT imaging. Furthermore, before the administration of ${ }^{123}$ I-labeled radioligands, thyroid protection is part of the protocol, which is not the case for ${ }^{18} \mathrm{~F}$ or ${ }^{11} \mathrm{C}$ DAT-PET radioligands.

It can be argued that the data acquisition itself is shorter for DAT-SPECT (20 min) than DAT-PET (minimum 


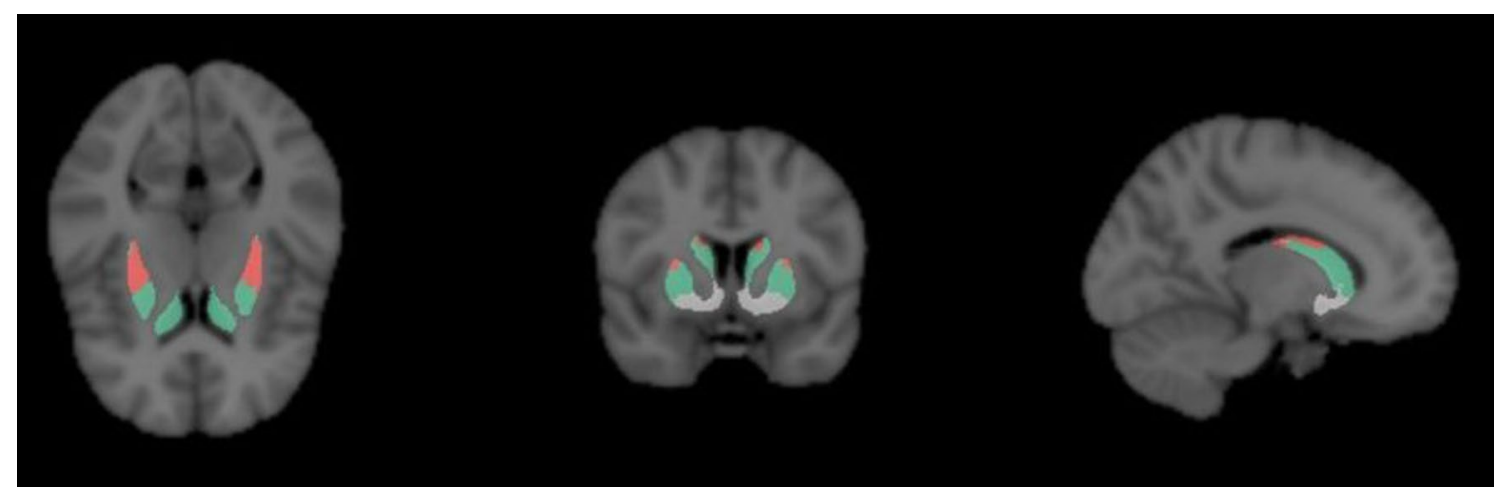

Fig. 1 Functional subdivisions of striatum. Template as created by Tziortzi et al. (https://fsl.fmrib.ox.ac.uk/fsl/fslwiki/Atlases/striatumconn), overlaid on T1 MRI sequences. Red: sensorimotor striatum; green: associative striatum; gray: limbic striatum

65 min). However, recent studies with the DAT-PET radioligand $\left[{ }^{18} \mathrm{~F}\right] \mathrm{FE}-\mathrm{PE} 2 \mathrm{I}$ have concluded that a shortened, nondynamic, acquisition time window can be used for simplified quantification (specific binding ratio, SBR). SBR measured during the early time window (16.5-42 min after radioligand injection) was most reliable and well correlated to full quantification (binding potential). SBR measured during a later pseudo-equilibrium time window was sufficient for the purpose of discriminating PS from healthy controls [23, 36-38]. This makes $\left[{ }^{18} \mathrm{~F}\right] \mathrm{FE}-\mathrm{PE} 2 \mathrm{I}$ additionally suitable for static image acquisition, which is necessary for a clinical setting.

With regards to radioligand production, the short halflife of ${ }^{11} \mathrm{C}$ (20.33 min) restricts its use to facilities that can produce the radioligand in-house. The longer half-life of ${ }^{18} \mathrm{~F}$ (109.77 min) makes transportation of the radioligand from an external production site to the local hospitals possible. At the same time, more nuclear molecular imaging centers have GMP facilities, so local production is increasingly possible. If the radiopharmaceutical production of ${ }^{18} \mathrm{~F}$-labeled DAT radioligands is optimized so that a similar amount of radioactivity as $\left[{ }^{18} \mathrm{~F}\right] \mathrm{FDG}$ can be produced, then several injection doses can be prepared from one single batch, lowering production costs.

It is well acknowledged that the SPECT systems are less expensive than PET systems, and that costs for the production of SPECT radiopharmaceuticals are lower than PET radiopharmaceuticals. This gap, however, can be made smaller if the use and production of PET radioligands can be optimized.

Since DAT-SPECT has been widely implemented in clinical care and is more available than DAT-PET, this has created big DAT-SPECT sample collections for studies (https://www.ppmi-info.org/publications-presentati ons/publications/, [29, 39-41]) and machine learning and analysis automatization [42-44]. If DAT-PET were to find its way into the clinic, the same increased cohort sizes could be achieved, and lack of the expected correlations in DAT-SPECT studies could be re-evaluated with the possibility of more specific regions of interest.

\section{Flexibility}

Modern PET systems and acquisition give rise to the possibility of multimodal imaging within the same imaging session. This is efficient and can provide a better estimation of the underlying pathophysiological processes $[4$, 45].

\section{PET-MRI}

With the rise of PET-MRI systems, both PET and structural MRI measurements can be conducted at the same time, simplifying co-registration, saving time, and reducing radiation burden. In the clinical setting, combined PET-MRI might simplify the diagnostic workflow. From the pathophysiological point of view, multimodality imaging makes it possible to study different imaging markers at the same time. For example, simultaneous assessment of neuromelanin in the $\mathrm{SN}$, as biomarker of dopaminergic cell body integrity, and DAT in the striatum, as biomarker of terminal function, will help to understand how they relate [46, 47]. Martín-Bastida et al. [34] found neuromelanin to correlate with disease duration and DAT with UPDRS motor score, concluding neuromelanin might reflect the underlying pathophysiological process, whereas DAT represents the functional state of the dopaminergic system [48]. Nigrosome imaging [49, 50] using $\mathrm{T} 2 *$ and susceptibility weighted imaging MRI sequences could be another SN MR-biomarker of interest to combine with DAT imaging. 


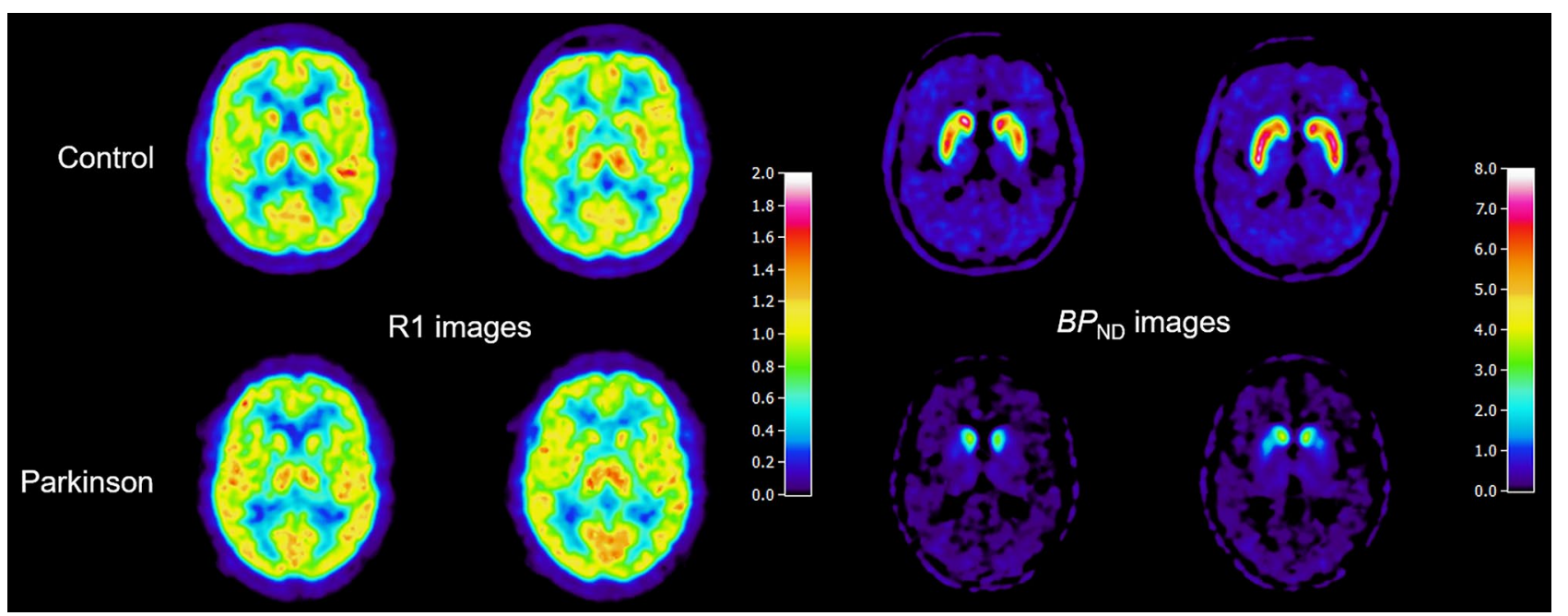

Fig. 2 Parametric imaging of relative blood flow (R1) and nondisplaceable binding potential $\left(B P_{N D}\right)$ of $\left[{ }^{18} \mathrm{~F}\right] \mathrm{FE}-\mathrm{PE} 2 \mathrm{I}$. These parametric images are derived from the same dynamic imaging session

Further validation of the appropriate reference tissue and attenuation correction methods for DAT imaging with the new PET-MRI systems, however, is needed [38, 51-53].

\section{Early perfusion}

Considering the functional coupling between perfusion and metabolism, early perfusion images have been considered as a potential alternative to $\left[{ }^{18} \mathrm{~F}\right] \mathrm{FDG}$, as in the case of tau and amyloid imaging [54-57]. Where DAT imaging cannot provide further distinction between PD and the different atypical Parkinson syndromes, FDG-PET has been more successful. Specific visual patterns of hypometabolism have been observed in MSA, PSP, and CBD. In addition, principal component analysis of FDG-PET has yielded several disease-specific patterns as well (such as Parkinson's diseaserelated pattern [PDRP], MSA pattern, and PDP pattern) that have helped differential diagnoses [58-60]. A study with $\left[{ }^{18} \mathrm{~F}\right]$ FP-CIT has shown that relative blood perfusion and DAT availability can be measured in the same imaging session [61, 62]. In addition, a study performed with [ $\left.{ }^{11} \mathrm{C}\right] \mathrm{PE} 2 \mathrm{I}$ in MSA and PD patients has shown that parametric images of R1 (relative blood flow) and binding potential (DAT availability) can be obtained from the same imaging session [63] and can help the differential diagnosis. Parametric images of R1 can also be obtained with $\left[{ }^{18} \mathrm{~F}\right] \mathrm{FE}-\mathrm{PE} 2 \mathrm{I}$ (Fig. 2).

\section{Multiple pre-synaptic dopaminergic targets}

Regarding imaging of the pre-synaptic dopaminergic system, more targets exist than just the DAT. PET radioligands for the vesicular monoamine transporter (VMAT2) or aromatic
(93 min). Images show lower striatal metabolism and binding potential in the PD patient compared to the healthy control. Courtesy of My Jonasson and Mark Lubberink, Uppsala University

1-amino acid decarboxylase (AADC) are available and widely used as imaging tools for PD [4, 6, 64]. SPECT tracers for the same targets are not available. Hence, PET provides the unique opportunity to image different pre-synaptic dopaminergic targets in the same subject, with the possibility to study their respective roles in pre-synaptic dopaminergic functioning and integrity in subjects with parkinsonian diseases. Multitracer imaging of pre-synaptic terminals can, therefore, give insight in the possible functional adaptation of each target in the striatum (3) and the rate of progression of different dopaminergic markers in PD (4).

\section{Conclusion}

DAT-SPECT is well established for differentiating degenerative from non-degenerative parkinsonism. SPECT is less expensive than PET and currently more available, making it still preferable for clinical studies aiming to have big sample sizes. However, now that practical PET disadvantages have been overcome, DAT-PET can be considered worldwide for a more active role in the clinic, to facilitate a more comprehensive understanding and assessment of PD.

Author contributions VSK: literature search and review, manuscript writing and editing. AV: content planning, literature review, and manuscript editing.

Funding VSK's income has been supported by KID funds for doctoral students at Karolinska Institutet and by funds from the Swedish Science Council. The development and validation of $\left[{ }^{18} \mathrm{~F}\right] \mathrm{FE}-\mathrm{PE} 2 \mathrm{I}$ as imaging marker in PD has been supported by a grant from the Swedish 
Foundation for Strategic Research, a grant from AstraZeneca Translational Science Centre at Karolinska Institutet, a grant from the Swedish Foundation for Parkinson's Research, a private donation, and a grant from the Swedish Science Council. Karolinska Institutet (2016-00186), Åhlén-stiftelsen (SE) (mA4/h17), Vetenskapsrådet (SE) (2016-02607). Open access funding provided by Karolinska Institute.

\section{Compliance with ethical standards}

Conflict of interest The PET radioligand $\left[{ }^{18} \mathrm{~F}\right] \mathrm{FE}-\mathrm{PE} 2 \mathrm{I}$ has been developed at Karolinska Institutet in the research group of the authors.

Humand and animal rights This article is a review and does not contain new studies on human or animal subjects performed by any of the authors.

Open Access This article is licensed under a Creative Commons Attribution 4.0 International License, which permits use, sharing, adaptation, distribution and reproduction in any medium or format, as long as you give appropriate credit to the original author(s) and the source, provide a link to the Creative Commons licence, and indicate if changes were made. The images or other third party material in this article are included in the article's Creative Commons licence, unless indicated otherwise in a credit line to the material. If material is not included in the article's Creative Commons licence and your intended use is not permitted by statutory regulation or exceeds the permitted use, you will need to obtain permission directly from the copyright holder. To view a copy of this licence, visit http://creativecommons.org/licenses/by/4.0/.

\section{References}

1. Merchant KM, Cedarbaum JM, Brundin P et al (2019) A proposed roadmap for Parkinson's disease proof of concept clinical trials investigating compounds targeting alpha-synuclein. J Parkinsons Dis 9:31-61

2. Uzuegbunam BC, Librizzi D, Hooshyar Yousefi B (2020) PET Radiopharmaceuticals for Alzheimer's disease and Parkinson's disease diagnosis, the current and future landscape. Molecules 25:977. https://doi.org/10.3390/molecules 25040977

3. Lee CS, Samii A, Sossi V et al (2000) In vivo positron emission tomographic evidence for compensatory changes in presynaptic dopaminergic nerve terminals in Parkinson's disease. Ann Neurol. https://doi.org/10.1002/1531-8249(200004)47:4<493:AIDANA13>3.0.CO;2-4

4. Nandhagopal R, Kuramoto L, Schulzer M et al (2011) Longitudinal evolution of compensatory changes in striatal dopamine processing in Parkinson's disease. Brain. https://doi.org/10.1093/ brain/awr233

5. Varrone A, Halldin C (2014) Human brain imaging of dopamine transporters. In: Imaging of the human brain in health and disease. Neuroscience-Net, Eds. Philip Seeman and Bertha Madras

6. Kaasinen V, Kankare T, Joutsa J, Vahlberg T (2019) Presynaptic striatal dopaminergic Function in atypical parkinsonism: a metaanalysis of imaging studies. J Nucl Med. 60(12):1757-1763. https://doi.org/10.2967/jnumed.119.227140

7. Sakakibara S, Hashimoto R, Katayama T et al (2020) Longitudinal change of DAT SPECT in Parkinson's disease and multiple system atrophy. J Parkinsons Dis 10:123-130. https://doi.org/10.3233/ JPD-191710

8. Graebner AK, Tarsy D, Shih LC et al (2016) Clinical impact of 123I-ioflupane SPECT (DaTscan) in a movement disorder center. Neurodegener Dis 17:38-43. https://doi.org/10.1159/000447561
9. Sadasivan S, Friedman JH (2015) Experience with DaTscan at a tertiary referral center. Parkinson Relat Disord. https://doi. org/10.1016/j.parkreldis.2014.10.022

10. Maiti B, Perlmutter JS (2018) PET Imaging in movement disorders. Semin Nucl Med 48:513-524

11. Buchert R, Buhmann C, Apostolova I et al (2019) Nuclear imaging in the diagnosis of clinically uncertain parkinsonian syndromes. Dtsch Aerzteblatt Online. https://doi.org/10.3238/arzte bl.2019.0747

12. Palermo G, Ceravolo R (2019) Molecular imaging of the dopamine transporter. Cells. 8(8):872. https://doi.org/10.3390/cells 8080872

13. Morbelli S, Esposito G, Arbizu J et al (2020) EANM practice guideline/SNMMI procedure standard for dopaminergic imaging in Parkinsonian syndromes 1.0. Eur J Nucl Med Mol Imaging. https://doi.org/10.1007/s00259-020-04817-8

14. Djang DS, Janssen MJ, Bohnen N et al (2012) SNM practice guideline for dopamine transporter imaging with 123I-ioflupane SPECT 1.0. J Nucl Med. 53(1):154-163. https://doi.org/10.2967/ jnumed.111.100784

15. Menéndez-González M, Tavares F, Zeidan N et al (2014) Diagnoses behind patients with hard-to-classify tremor and normal DaT-SPECT: A clinical follow up study. Front Aging Neurosci. https://doi.org/10.3389/fnagi.2014.00056

16. Stephenson D, Hill D, Cedarbaum JM et al (2019) The qualification of an enrichment biomarker for clinical trials targeting early stages of Parkinson's disease. J Parkinson Dis. https://doi. org/10.3233/JPD-191648

17. Marshall VL, Reininger CB, Marquardt M et al (2009) Parkinson's disease is overdiagnosed clinically at baseline in diagnostically uncertain cases: A 3-year European multicenter study with repeat [123I]FP-CIT SPECT. Mov Disord. https://doi.org/10.1002/ mds. 22108

18. Hutton BF, Occhipinti M, Kuehne A et al (2018) Development of clinical simultaneous SPECT/MRI. Br J Radiol. 91(1081):20160690. https://doi.org/10.1259/bjr.20160690

19. Varrone A, Halldin C (2010) Molecular imaging of the dopamine transporter. J Nucl Med. 51(9):1331-1334. https://doi. org/10.2967/jnumed.109.065656

20. Varrone A, Halldin C (2012) New developments of dopaminergic imaging in Parkinson's disease. Q J Nucl Med Mol Imaging. 56(1):68-82

21. Marek KL, Seibyl JP, Zoghbi SS et al (1996) [123I] beta-CIT/ SPECT imaging demonstrates bilateral loss of dopamine transporters in hemi-Parkinson's disease. Neurology 46(1):231-237. https://doi.org/10.1212/wnl.46.1.231

22. Booij J, Habraken JB, Bergmans P et al (1998) Imaging of dopamine transporters with iodine-123-FP-CIT SPECT in healthy controls and patients with Parkinson's disease. J Nucl Med 39:1879-1884

23. Jakobson Mo S, Axelsson J, Jonasson L et al (2018) Dopamine transporter imaging with [18F]FE-PE2I PET and [123I]FPCIT SPECT - a clinical comparison. EJNMMI Res. https://doi. org/10.1186/s13550-018-0450-0

24. Lee I, Kim JS, Park JY et al (2018) Head-to-head comparison of 18 F-FP-CIT and 123 I-FP-CIT for dopamine transporter imaging in patients with Parkinson's disease: A preliminary study. Synapse. https://doi.org/10.1002/syn.22032

25. Badoud S, Van De Ville D, Nicastro N et al (2016) Discriminating among degenerative parkinsonisms using advanced 123I-ioflupane SPECT analyses. NeuroImage Clin. https://doi.org/10.1016/j. nicl.2016.07.004

26. Mittal BR, Sood A, Shukla J et al (2018) 99m Tc-TRODAT-1 SPECT/CT imaging as a complementary biomarker in the 
diagnosis of parkinsonian syndromes. Nucl Med Commun. https ://doi.org/10.1097/MNM.0000000000000802

27. Matesan M, Gaddikeri S, Longfellow K et al (2018) I-123 DaTscan SPECT brain imaging in Parkinsonian syndromes: utility of the putamen-to-caudate ratio. J Neuroimaging. https://doi. org/10.1111/jon. 12530

28. Fazio P, Svenningsson P, Cselényi Z et al (2018) Nigrostriatal dopamine transporter availability in early Parkinson's disease. Mov Disord 33:592-599. https://doi.org/10.1002/mds.27316

29. Caspell-Garcia C, Simuni T, Tosun-Turgut D et al (2017) Multiple modality biomarker prediction of cognitive impairment in prospectively followed de novo Parkinson disease. PLoS ONE. https://doi.org/10.1371/journal.pone.0175674

30. Picillo M, Santangelo G, Erro R et al (2017) Association between dopaminergic dysfunction and anxiety in de novo Parkinson's disease. Parkinsonism Relat Disord. https://doi.org/10.1016/j.parkr eldis.2017.02.010

31. Joel D, Weiner I (2000) The connections of the dopaminergic system with the striatum in rats and primates: an analysis with respect to the functional and compartmental organization of the striatum. Neuroscience. 96(3):451-474. https://doi.org/10.1016/ s0306-4522(99)00575-8

32. Tziortzi AC, Searle GE, Tzimopoulou $S$ et al (2011) Imaging dopamine receptors in humans with [11C]-(+)-PHNO: dissection of D3 signal and anatomy. Neuroimage. https://doi.org/10.1016/j. neuroimage.2010.06.044

33. Tziortzi AC, Haber SN, Searle GE et al (2014) Connectivity-based functional analysis of dopamine release in the striatum using diffusion-weighted MRI and positron emission tomography. Cereb Cortex. https://doi.org/10.1093/cercor/bhs397

34. Martín-Bastida A, Lao-Kaim NP, Roussakis AA et al (2019) Relationship between neuromelanin and dopamine terminals within the Parkinson's nigrostriatal system. Brain 142:2023-2036. https ://doi.org/10.1093/brain/awz120

35. Oh M, Kim JS, Kim JY et al (2012) Subregional patterns of preferential striatal dopamine transporter loss differ in Parkinson disease, progressive supranuclear palsy, and multiple-system atrophy. J Nucl Med. https://doi.org/10.2967/jnumed.111.095224

36. Ikoma Y, Sasaki T, Kimura Y et al (2015) Evaluation of semiquantitative method for quantification of dopamine transporter in human PET study with 18F-FE-PE2I. Ann Nucl Med. https://doi. org/10.1007/s12149-015-0993-3

37. Sonni I, Fazio P, Schain M et al (2016) optimal acquisition time window and simplified quantification of dopamine transporter availability using 18F-FE-PE2I in healthy controls and Parkinson disease patients. J Nucl Med 57:1529-1534. https://doi. org/10.2967/jnumed.115.171231

38. Delva A, Van Weehaeghe D, van Aalst J et al (2019) Quantification and discriminative power of 18F-FE-PE2I PET in patients with Parkinson's disease. Eur J Nucl Med Mol Imaging. https:// doi.org/10.1007/s00259-019-04587-y

39. Parkinson Progression Marker Initiative (2011) The Parkinson Progression Marker Initiative (PPMI). Prog Neurobiol. 95(4):629 635. https://doi.org/10.1016/j.pneurobio.2011.09.005

40. Marek K, Chowdhury S, Siderowf A et al (2018) The Parkinson's progression markers initiative (PPMI) — establishing a PD biomarker cohort. Ann Clin Transl Neurol. https://doi.org/10.1002/ acn3.644

41. Hinkle JT, Perepezko K, Mills KA et al (2018) Dopamine transporter availability reflects gastrointestinal dysautonomia in early Parkinson disease. Parkionson Relat Disord. https://doi. org/10.1016/j.parkreldis.2018.08.010

42. Prashanth R, Dutta Roy S, Mandal PK, Ghosh S (2017) Highaccuracy classification of Parkinson's disease through shape analysis and surface fitting in 123I-Ioflupane SPECT imaging. IEEE J Biomed Heal Inf. https://doi.org/10.1109/JBHI.2016.2547901
43. Wenzel M, Milletari F, Krüger J et al (2019) Automatic classification of dopamine transporter SPECT: deep convolutional neural networks can be trained to be robust with respect to variable image characteristics. Eur J Nucl Med Mol Imaging. https://doi. org/10.1007/s00259-019-04502-5

44. Taylor JC, Fenner JW (2017) Comparison of machine learning and semi-quantification algorithms for (I123)FP-CIT classification: the beginning of the end for semi-quantification? EJNMMI Phys. https://doi.org/10.1186/s40658-017-0196-1

45. Südmeyer M, Antke C, Zizek T et al (2011) Diagnostic accuracy of combined FP-CIT, IBZM, and MIBG scintigraphy in the differential diagnosis of degenerative parkinsonism: A multidimensional statistical approach. J Nucl Med. https://doi.org/10.2967/ jnumed.110.086959

46. Isaias IU, Trujillo P, Summers P et al (2016) Neuromelanin imaging and dopaminergic loss in parkinson's disease. Front Aging Neurosci. https://doi.org/10.3389/fnagi.2016.00196

47. Kawaguchi H, Shimada H, Kodaka F et al (2016) Principal component analysis of multimodal neuromelanin mri and dopamine transporter pet data provides a specific metric for the Nigral. PLoS ONE. https://doi.org/10.1371/journal.pone.0151191

48. Honkanen EA, Saari L, Orte K et al (2019) No link between striatal dopaminergic axons and dopamine transporter imaging in Parkinson's disease. Mov Disord 34:1562-1566. https://doi. org/10.1002/mds. 27777

49. Mahlknecht P, Krismer F, Poewe W, Seppi K (2017) Meta-analysis of dorsolateral nigral hyperintensity on magnetic resonance imaging as a marker for Parkinson's disease. Mov Disord 32:619-623. https://doi.org/10.1002/mds.26932

50. Pavese N, Tai YF (2018) Nigrosome imaging and neuromelanin sensitive MRI in diagnostic evaluation of Parkinsonism. Mov Disord Clin Pract. https://doi.org/10.1002/mdc3.12590

51. Sousa JM, Appel L, Engström M et al (2018) Evaluation of zeroecho-time attenuation correction for integrated PET/MR brain imaging - comparison to head atlas and 68Ge-transmission-based attenuation correction. EJNMMI Phys. https://doi.org/10.1186/ s40658-018-0220-0

52. Tondo G, Esposito M, Dervenoulas G, Wilson H, Politis M, Pagano G (2019) Hybrid PET-MRI Applications in Movement Disorders. Int Rev Neurobiol. 144:211-257. https://doi. org/10.1016/bs.irn.2018.10.003

53. Mackewn JE, Stirling J, Jeljeli S et al (2020) Practical issues and limitations of brain attenuation correction on a simultaneous PETMR scanner. EJNMMI Phys. https://doi.org/10.1186/s40658-02000295-X

54. Hsiao IT, Huang CC, Hsieh CJ et al (2012) Correlation of earlyphase 18F-florbetapir (AV-45/Amyvid) PET images to FDG images: Preliminary studies. Eur J Nucl Med Mol Imaging. https ://doi.org/10.1007/s00259-011-2051-2

55. Meyer PT, Hellwig S, Amtage F et al (2011) Dual-biomarker imaging of regional cerebral amyloid load and neuronal activity in dementia with PET and11C-Labeled Pittsburgh compound B. J Nucl Med. https://doi.org/10.2967/jnumed.110.083683

56. Tiepolt S, Hesse S, Patt M et al (2016) Early [18F]florbetaben and $[11 \mathrm{C}] \mathrm{PiB}$ PET images are a surrogate biomarker of neuronal injury in Alzheimer's disease. Eur J Nucl Med Mol Imaging. https ://doi.org/10.1007/s00259-016-3353-1

57. Daerr S, Brendel M, Zach C et al (2017) Evaluation of early-phase $[18 \mathrm{~F}]$-florbetaben PET acquisition in clinical routine cases. NeuroImage Clin. https://doi.org/10.1016/j.nicl.2016.10.005

58. Juh R, Kim J, Moon D et al (2004) Different metabolic patterns analysis of Parkinsonism on the 18F-FDG PET. Eur J Radiol. https ://doi.org/10.1016/S0720-048X(03)00214-6

59. Eckert T, Tang C, Ma Y et al (2008) Abnormal metabolic networks in atypical parkinsonism. Mov Disord. https://doi.org/10.1002/ mds. 21933 
60. Tang CC, Poston KL, Eckert T et al (2010) Differential diagnosis of parkinsonism: a metabolic imaging study using pattern analysis. Lancet Neurol. https://doi.org/10.1016/S1474-4422(10)70002 $-8$

61. Hong C-M, Ryu H-S, Ahn B-C (2018) Early perfusion and dopamine transporter imaging using $18 \mathrm{~F}-\mathrm{FP}-\mathrm{CIT} \mathrm{PET} / \mathrm{CT}$ in patients with parkinsonism. Am J Nucl Med Mol Imaging 8:360-372

62. Jin S, Oh M, Oh SJ et al (2017) Additional value of early-phase 18F-FP-CIT PET image for differential diagnosis of atypical Parkinsonism. Clin Nucl Med. https://doi.org/10.1097/RLU.00000 00000001474

63. Appel L, Jonasson M, Danfors T et al (2015) Use of 11C-PE2I PET in differential diagnosis of parkinsonian disorders. J Nucl Med 56:234-242. https://doi.org/10.2967/jnumed.114.148619
64. Fu JF, Klyuzhin I, McKenzie J et al (2019) Joint pattern analysis applied to PET DAT and VMAT2 imaging reveals new insights into Parkinson's disease induced presynaptic alterations. NeuroImage Clin. https://doi.org/10.1016/j.nicl.2019.101856

Publisher's Note Springer Nature remains neutral with regard to jurisdictional claims in published maps and institutional affiliations. 